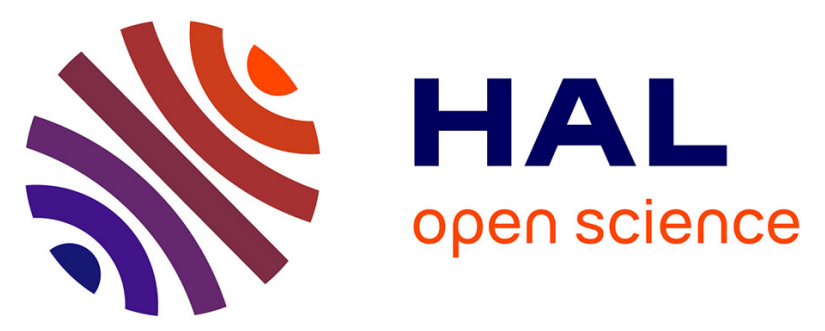

\title{
A complementary tool for management of disseminated Histoplasma capsulatum var. capsulatum infections in AIDS patients
}

Xavier Iriart, Denis Blanchet, Sandie Ménard, Rose-Anne Lavergne, Pamela Chauvin, Antoine Adenis, Sophie Cassaing, Judith Fillaux, Jean-François Magnaval, Magalie Pierre Demar, et al.

\section{To cite this version:}

Xavier Iriart, Denis Blanchet, Sandie Ménard, Rose-Anne Lavergne, Pamela Chauvin, et al.. A complementary tool for management of disseminated Histoplasma capsulatum var. capsulatum infections in AIDS patients: A complementary tool for histoplasmosis management. International Journal of Medical Microbiology, 2014, 304 (8), pp.1062-1065. 10.1016/j.ijmm.2014.07.016 . inserm-01065096

\section{HAL Id: inserm-01065096 https://www.hal.inserm.fr/inserm-01065096}

Submitted on 17 Sep 2014

HAL is a multi-disciplinary open access archive for the deposit and dissemination of scientific research documents, whether they are published or not. The documents may come from teaching and research institutions in France or abroad, or from public or private research centers.
L'archive ouverte pluridisciplinaire HAL, est destinée au dépôt et à la diffusion de documents scientifiques de niveau recherche, publiés ou non, émanant des établissements d'enseignement et de recherche français ou étrangers, des laboratoires publics ou privés. 


\title{
A complementary tool for management of disseminated Histoplasma capsulatum var. capsulatum infections in AIDS patients
}

\author{
Xavier IRIART ${ }^{1,6}$, Denis BLANCHET ${ }^{2}$, Sandie MENARD ${ }^{6}$, Rose-Anne LAVERGNE ${ }^{1,6}$, \\ Pamela CHAUVIN ${ }^{1}$, Antoine ADENIS ${ }^{4}$, Sophie CASSAING ${ }^{1}$, Judith FILLAUX ${ }^{1}$, Jean- \\ François MAGNAVAL ${ }^{1}$, Magalie DEMAR ${ }^{2,5}$, Bernard CARME $^{2,5}$, Marie-Hélène \\ BESSIERES ${ }^{1}$, Pierre COUPPIE ${ }^{3,5}$, Mathieu NACHER ${ }^{4,5}$, Antoine BERRY $^{1,6}$ and \\ Christine AZNAR ${ }^{2,5}$

\footnotetext{
${ }^{1}$ Service de Parasitologie-Mycologie, Centre Hospitalier Universitaire de Toulouse, Toulouse, France ${ }^{2}$ Laboratoire Hospitalier et Universitaire Parasitologie Mycologie, ${ }^{3}$ Service de dermatologie, ${ }^{4}$ CIC-EC AntillesGuyane, CIE 802 Inserm, Centre Hospitalier de Cayenne, BP 6006, 97300 Cayenne, Guyane française

${ }^{5}$ Equipe EA 3593 Épidémiologie des Parasitoses et Mycoses Tropicales, Université Antilles Guyane, Campus Saint Denis, Cayenne, French Guiana

${ }^{6}$ INSERM UMR1043/CNRS UMR5282/Université de Toulouse UPS, Centre de Physiopathologie de Toulouse
} \\ Purpan (CPTP), Toulouse, F-31300, France
}

Reprints or correspondence: Christine Aznar (Email: christine.aznar1@wanadoo.fr), Laboratoire Hospitalier et Universitaire Parasitologie Mycologie, CH Andrée Rosemon, Cayenne 97306, Guyane Française or Xavier Iriart (Email: iriart.x @ chu-toulouse.fr), Service de Parasitologie-Mycologie, Centre Hospitalier Universitaire de Toulouse, Hôpital Purpan, Institut Fédératif de biologie (IFB), 330 avenue de Grande Bretagne, TSA 40031, 31059 Toulouse Cedex 9, France.

RUNNING TITLE: A complementary tool for histoplasmosis management

\begin{abstract}
In South America, disseminated histoplasmosis due to Histoplasma capsulatum var. capsulatum (H. capsulatum), is a severe and frequent opportunistic infection in AIDS patients. In areas outside the USA where specific-Histoplasma antigen detection is not available, the diagnosis is difficult. With the galactomannan antigen (GM) detection, a test commonly used for invasive aspergillosis diagnosis, there is a cross-reactivity with $H$. capsulatum that can be helpful for the diagnosis of histoplasmosis. The aim of this study was to evaluate the GM detection for the diagnosis of disseminated histoplasmosis in AIDS patients. The performance of the GM detection was evaluated with serum collected in French Guiana where $H$. capsulatum is highly endemic. Sera from AIDS patients with disseminated histoplasmosis occurring from 2002 to 2009 and from control HIV-positive patients without histoplasmosis were tested with the GM detection and Histoplasma-specific antibody detection (IEP). In 39 AIDS patients with proven disseminated histoplasmosis, the sensitivity of the Histoplasma IEP was only $35.9 \%$ and was linked to the TCD4+ lymphocyte level. For the GM detection, the sensitivity (Se) was $76.9 \%$ and specificity ( $\mathrm{Sp}$ ) was $100 \%$ with the recommended threshold for aspergillosis diagnosis (0.5). The test was more efficient with a threshold of 0.4 (Se: 0.82 [95\% CI: 0.66-0.92], Sp: 1.00 [95\% CI: 0.86-1.00], LR+: >10, LR-: 0.18 ). This study confirms that the GM detection can be a surrogate marker for the diagnosis of disseminated histoplasmosis in AIDS patients in endemic areas where Histoplasma EIA is not available.
\end{abstract}


KEYWORDS : histoplasmosis; AIDS; Histoplasma capsulatum; Diagnosis; Platelia; galactomannan; antibody; cross-reactivity

\section{INTRODUCTION}

Histoplasmosis caused by Histoplasma capsulatum var. capsulatum (H. capsulatum) is endemic in the United States, in several countries of Central and South America and in scattered areas of Asia and Africa (Kauffman, 2007). Disseminated histoplasmosis is a severe illness that occurs almost exclusively in immunosuppressed patients, particularly in patients with acquired immunodeficiency syndrome (AIDS). In French Guiana, histoplasmosis is the most frequent opportunistic infection in HIV-infected patients and the first cause of AIDSrelated death (Couppie et al., 2004; Huber et al., 2008; Lewden et al., 2004). Even if isolation of Histoplasma from cultures is the reference procedure for histoplasmosis diagnosis (Kauffman, 2008), it can take weeks and is positive in only 50-70\% of cases (Sathapatayavongs et al., 1983). Serological methods based on specific antibody detection can be performed rapidly but usually give false negative results in AIDS patients (Tobon et al., 2005). In this context, circulating specific-Histoplasma antigen detection (Histoplasma EIA) represents a useful option for diagnosis but this method is often unavailable in the majority of endemic areas outside the USA (Connolly et al., 2007; Hage et al., 2011).

The Platelia Aspergillus enzyme immunoassay (EIA) is a ready-to-use test which detects galactofuranose-containing side chains of galactomannan. This test is commonly used for the diagnosis of invasive aspergillosis, particularly in solid organ transplant recipients or patients with haematological malignancies (Aquino et al., 2007), but cross-reactivity exists with many other fungi (Aquino et al., 2007; Dalle et al., 2005; Desoubeaux et al., 2014; Giacchino et al., 2006; Huang et al., 2007; Van Der Veer et al., 2012; Xavier et al., 2009) in particular $H$. capsulatum (Narreddy and Chandrasekar, 2008; Wheat et al., 2007). Galactomannan antigen (GM) detection can lead to a false-positive diagnosis of aspergillosis in these immunocompromised patients who have histoplasmosis (Jones et al., 2009; Vergidis et al., 2012). In areas outside the USA where Histoplasma EIA is not available, this cross-reactivity with the galactomannan antigen could be helpful for the diagnosis of disseminated histoplasmosis (Pineau et al., 2010; Ranque et al., 2007; Riviere et al., 2012). GM detection could be particularly interesting in HIV-infected patients because of the low incidence of invasive aspergillosis in this population compared to patients with organ transplantation or hematological malignancies (Desoubeaux et al., 2014).

Given the rarity of histoplasmosis in Europe, preliminary studies on GM detection for this diagnosis were only conducted on a limited number of samples (Ranque et al., 2007). Thus, the aim of our study was to evaluate more widely the GM detection for the diagnosis of disseminated histoplasmosis in AIDS patients. This evaluation was carried out in a $H$. capsulatum endemic area (French Guiana) to obtain a larger cohort of patients.

\section{PATIENTS AND METHODS}

\section{Study population}

Disseminated histoplasmosis cases occurring in AIDS patients followed in the Cayenne hospital (French Guiana) were selected from 2002 to 2009. Among this cohort, all patients for whom sera were collected at the time of diagnosis of histoplasmosis $( \pm 10$ days=baseline $)$ and available for analysis (stored at $-20^{\circ} \mathrm{C}$ ) were included in the study. HIV-patients were 
considered as histoplasmosis cases, if there was microbiological evidence of Histoplasma infection (positive direct examination and/or culture and/or polymerase chain reaction (Simon et al., 2010) for Histoplasma capsulatum). Thirty HIV-positive patients who had never been in an endemic area for Histoplasma were included as negative controls. For these controls, no critical antibiotics (Piperacillin-tazobactam), polyvalent immunoglobulins or dialysis were present when GM detection was performed.

\section{GM detection and Histoplasma-specific antibody detection}

Sera stored at $-20^{\circ} \mathrm{C}$ were tested with the Platelia Aspergillus EIA (BioRad, France) in accordance with the manufacturer's specifications. Briefly, after a pretreatment $(6 \mathrm{~min}$, $120^{\circ} \mathrm{C}$ ), $50 \mu \mathrm{l}$ of serum was added to $50 \mu \mathrm{l}$ of conjugate before being incubated at $37^{\circ} \mathrm{C}$ for 90 min. After washing, chromogen substrate solution was added and the plates were darkincubated for 30min. After the addition of stop solution, the optical density (OD) was determined at $450 \mathrm{~nm}$ (reference filter $620 / 630 \mathrm{~nm}$ ). Samples were considered as positive when the galactomannan index (GMI) was $\geq 0.5$ (De Pauw et al., 2008).

In parallel, an immunoelectrophoretic assay for Histoplasma-specific antibody detection (IEP) was performed according to manufacturer's instruction (Beckman Paragon, France). Briefly, $3 \mu \mathrm{L}$ of Histoplasma antigen (Laboratoire Méridian, France) were electrophoresed (20min, 100V; Beckman Paragon, France) on ready-to-use Hydragel-IEP-Plus gels (Sebia, France). After a 24h-incubation with serum, the gel was washed, placed under a press, stained with acid violet, washed again and finally dried out. The test was considered as positive if at least one precipitation line was detected by visual observation.

\section{Analysis and Statistical Methods}

Data were analyzed with SIGMA Stat software (2.03) using the Mann-Whitney rank sum test for a two-group comparison and the $\chi^{2}$ test for patient characteristic comparison. Relationships between two variables were analyzed by Spearman rank order correlation test. Values were reported as the median and interquartile range IQR [25\%; 75\%]. Analysis of Receiver Operator Characteristic (ROC) curves was performed to determine the cut-off for positivity. A comparison was considered statistically significant if the $p$ value was $\leq 0.05$.

\section{RESULTS}

\section{Patient Characteristics}

Between 2002 and 2009, 39 AIDS patients diagnosed for disseminated histoplasmosis and with an available concomitant serum were included in the study (Table 1). There was no significant difference in the median age or sex ratio between case and control patients. Diagnosis of histoplasmosis was obtained mainly on hematology $(48.7 \%)$ or digestive (28.2\%) samples. Thirty seven patients $(94.9 \%)$ had a positive culture for Histoplasma capsulatum. For two patients, a rapid and extensive development of Candida albicans on the culture did not allow the growth of Histoplasma but PCR and direct examination were both positive, confirming the diagnosis of histoplasmosis. All the index case and control patients were negative for the diagnosis of Aspergillus infection (culture and anti-Aspergillus antibody detection).

\section{Anti-Histoplasma antibody detection}

For AIDS-patients with disseminated histoplasmosis, the sensitivity (Se) of Histoplasma IEP was only 35.9\% [95\% CI: 21.7-52.8] while the specificity (Sp) was 100\% [95\% CI: 85.9-100] (Table 1). The sensitivity was linked to the TCD4+ lymphocyte level as the counts of these cells was statistically higher in patients with positive Histoplasma-specific antibody detection 
than in patients with negative serology $(26[5 ; 37]$ vs 84 [77; 90]) ( $\mathrm{p}=0.007$; Mann-Whitney rank sum test). On the contrary, there was no statistical difference for TCD8+ lymphocyte levels (data not shown).

\section{GM detection for diagnosis of histoplasmosis}

Galactomannan indexes (GMI) were significantly higher in HIV-positive patients with histoplasmosis compared to Histoplasma-uninfected ones (Table 1). With the recommended threshold for invasive aspergillosis diagnosis (0.5), the sensitivity was $76.9 \%$ [95\% confidence intervals (95\% CI): 60.3-88.3] and the specificity was 100\% [95\% CI: 85.9-100] for histoplasmosis diagnosis. The TCD4+ or TCD8+ lymphocytes counts were not statistically different between groups of patients with positive or negative GMI (data not shown). Moreover, the GMI level was not correlated with TCD4+ or TCD8+ lymphocyte counts in HIV-positive patients with histoplasmosis (data not shown).

The area under the curve was 0.963 on the ROC curve (Fig. 1). Two other thresholds $(0.4$ and 0.35 ) appeared to be potentially more interesting than the recommended threshold 0.5 , with sensitivities of $82.1 \%$ [95\% CI: 65.9-91.9] and 87.2\% [95\% CI: 71.8-95.1] and specificities of 100\% [95\% CI: 85.9-100] and 93.3\% [95\% CI: 76.5-98.8] respectively. Positive likelihood ratios (LR+) were 13.08 [95\% CI: 3.41-50.2], $+\infty$ [95\%CI: non-calculable], $+\infty$ [95\% CI: noncalculable] for thresholds of $0.35 ; 0.4 ; 0.5$ respectively and negative likelihood ratios (LR-) were 0.14 [95\% CI: $0.06-0.31$ ] 0.18 [95\% CI: $0.09-0.35$ ] and 0.23 [95\% CI: $0.13-0.41$ ] for thresholds of $0.35 ; 0.4 ; 0.5$ respectively.

Taking account of this information, the threshold 0.4 seems to be the most relevant (Se: $82.1 \%$ [95\% CI: 65.9-91.9], Sp: $100 \%$ [95\% CI: 85.9-100], LR+: $+\infty$ [95\% CI: noncalculable], LR-: 0.18 [95\% CI: 0.09-0.35]). Coupling galactomanann and anti-Histoplasma antibody detection did not significantly improve the overall diagnosis performance (Se: $82.1 \%$ [95\% CI: 65.9-91.9], Sp: 100\% [95\% CI: 85.9-100].

\section{DISCUSSION}

In areas outside the USA where Histoplasma EIA is not available, the diagnosis of disseminated histoplasmosis is often difficult to obtain in a timely manner consistent with the life-threatening character of this disease: culture takes a long time, direct examination is of poor sensitivity and Histoplasma-specific PCR is not available in most centers. As shown by others (Tobon et al., 2005; Wheat, 2006), we confirmed that detection of anti-Histoplasma specific antibodies had a low sensitivity (35.9\%) in AIDS patients. It is now well-established that CD4+T-cell depletion due to HIV was responsible for an IL-7-dependant alteration of Blymphocyte responses (Moir and Fauci, 2009). This phenomenon could explain the CD4+T cell-dependent decrease in anti-Histoplasma antibody production observed in our study among AIDS patients with histoplasmosis.

In contrast, we found that the GM detection performed well (Se: 76.9\%, Sp: 100\%). However, the threshold used for Aspergillus (0.5) could be lowered to 0.4 to increase the sensitivity (Se $82.1 \%$ ) without decreasing the specificity. Keeping the recommended threshold for aspergillosis diagnosis $(0.5)$ would lead to a decrease in sensitivity of $5.2 \%$. Unlike antibody levels, GMI were not influenced by TCD4+ lymphocyte counts. For GM detection, Ranque et $a l$. already reported a sensitivity of about $73 \%$ in 11 patients with pulmonary histoplasmosis and $100 \%$ in 6 HIV-positive patients (Ranque et al., 2007). Thus, in Histoplasma endemic areas where Histoplasma EIA is not available, this quick and easy-to-perform technique might be a powerful alternative for the diagnosis of disseminated histoplasmosis in HIV-positive patients. 
In two other studies (Wheat et al., 2007; Xavier et al., 2009), the sensitivity of the GM detection was about $48 \%$ and $67 \%$ in histoplasmosis diagnosis but these studies were not only limited to AIDS patients and the clinical presentation was not specified (e.g., fungemia versus non-disseminated disease). As the GM detection cross-reactivity seems to occur with high levels of Histoplasma antigens (Wheat et al., 2007), the sensitivity could be better in AIDS patients with disseminated disease because of a high Histoplasma burden (Ranque et al., 2007). For this reason, it seems to be essential to reserve the GM detection for disseminated histoplasmosis diagnosis in AIDS patients on the basis of epidemiological, clinical, and laboratory arguments. Moreover, as the incidence of invasive aspergillosis in HIV-positive patients is generally $<0.5 \%$ (Tong et al., 2009), the risk of misdiagnosis with aspergillosis is low. The positivity of the GM detection seems to be also very useful in the diagnosis and the monitoring of African histoplasmosis due to Histoplasma capsulatum var duboisii (Therby et al., 2014). Similarly, the cross-reactivity of the GM detection exists with Cryptocococcus (Dalle et al., 2005) but the incidence of this infection was lower in French Guiana with about 0.25 per 100 HIV/AIDS patients-years (Debourgogne et al., 2011). Nevertheless, an important limitation of this test concerns its significant cost. Moreover, the test requires a large number of controls which does not make its use consistent with small series. In South America, where histoplasmosis should be considered as neglected disease, the price of this test does not easily allow its use outside the rich countries.

GM detection could also be very helpful for histoplasmosis diagnosis outside endemic areas in HIV-positive travellers but the PPV and NPV should be reconsidered because of a lower prevalence of the disease in this context. However, the contribution of this test should be lower for histoplasmosis diagnosis in immunocompetent patients, especially compared to the detection of specific anti-Histoplasma antibodies that immunocompetent patients are able to synthesize.

The results of this study are somewhat limited by the retrospective design and the size of the cohort even if, to our knowledge, this is the largest study concerning GM detection specifically performed on AIDS patients with disseminated histoplasmosis. Moreover, the impact of serum storage at $-20^{\circ} \mathrm{C}$ is unknown on the performance of the $\mathrm{GM}$ detection test. However, it is usually believed that long-term storage may rather decrease galactomannan levels (Aquino et al., 2007) which would imply a higher sensitivity with fresh serum.

In conclusion, this study confirms that GM detection can be very helpful for the diagnosis of disseminated histoplasmosis in AIDS patients, particularly in endemic areas where Histoplasma EIA is not available.

\section{ACKNOWLEDGMENTS}

The authors gratefully acknowledge the technicians of Toulouse and Cayenne Hospital for technical assistance and John Woodley for the revision of the English.

Conflict of interest: The authors declare no conflicts of interest.

\section{REFERENCES}

Aquino, V.R., Goldani, L.Z., Pasqualotto, A.C., 2007. Update on the contribution of galactomannan for the diagnosis of invasive aspergillosis. Mycopathologia 163, 191-202.

Connolly, P.A., Durkin, M.M., Lemonte, A.M., Hackett, E.J., Wheat, L.J., 2007. Detection of histoplasma antigen by a quantitative enzyme immunoassay. Clin. Vaccine Immunol. 14, 1587-1591. 
Couppie, P., Sobesky, M., Aznar, C., Bichat, S., Clyti, E., Bissuel, F., El Guedj, M., Alvarez, F., Demar, M., Louvel, D., Pradinaud, R., Carme, B., 2004. Histoplasmosis and acquired immunodeficiency syndrome: a study of prognostic factors. Clin. Infect. Dis. 38, 134-138.

Dalle, F., Charles, P.E., Blanc, K., Caillot, D., Chavanet, P., Dromer, F., Bonnin, A., 2005. Cryptococcus neoformans Galactoxylomannan contains an epitope(s) that is cross-reactive with Aspergillus Galactomannan. J. Clin. Microbiol. 43, 2929-2931.

De Pauw, B., Walsh, T.J., Donnelly, J.P., Stevens, D.A., Edwards, J.E., Calandra, T., Pappas, P.G., Maertens, J., Lortholary, O., Kauffman, C.A., Denning, D.W., Patterson, T.F., Maschmeyer, G., Bille, J., Dismukes, W.E., Herbrecht, R., Hope, W.W., Kibbler, C.C., Kullberg, B.J., Marr, K.A., Munoz, P., Odds, F.C., Perfect, J.R., Restrepo, A., Ruhnke, M., Segal, B.H., Sobel, J.D., Sorrell, T.C., Viscoli, C., Wingard, J.R., Zaoutis, T., Bennett, J.E., 2008. Revised definitions of invasive fungal disease from the European Organization for Research and Treatment of Cancer/Invasive Fungal Infections Cooperative Group and the National Institute of Allergy and Infectious Diseases Mycoses Study Group (EORTC/MSG) Consensus Group. Clin. Infect. Dis. 46, 1813-1821.

Debourgogne, A., Iriart, X., Blanchet, D., Veron, V., Boukhari, R., Nacher, M., Carme, B., Aznar, C., 2011. Characteristics and specificities of Cryptococcus infections in French Guiana, 1998-2008. Med. Mycol. 49, 864-871.

Desoubeaux, G., Bailly, E., Chandenier, J., 2014. Diagnosis of invasive pulmonary aspergillosis: updates and recommendations. Med. Mal. Infect. 44, 89-101.

Giacchino, M., Chiapello, N., Bezzio, S., Fagioli, F., Saracco, P., Alfarano, A., Martini, V., Cimino, G., Martino, P., Girmenia, C., 2006. Aspergillus galactomannan enzyme-linked immunosorbent assay cross-reactivity caused by invasive Geotrichum capitatum. J. Clin. Microbiol. 44, 3432-3434.

Hage, C.A., Ribes, J.A., Wengenack, N.L., Baddour, L.M., Assi, M., McKinsey, D.S., Hammoud, K., Alapat, D., Babady, N.E., Parker, M., Fuller, D., Noor, A., Davis, T.E., Rodgers, M., Connolly, P.A., El Haddad, B., Wheat, L.J., 2011. A multicenter evaluation of tests for diagnosis of histoplasmosis. Clin. Infect. Dis. 53, 448-454.

Huang, Y.T., Hung, C.C., Hsueh, P.R., 2007. Aspergillus galactomannan antigenemia in penicilliosis marneffei. AIDS 21, 1990-1991.

Huber, F., Nacher, M., Aznar, C., Pierre-Demar, M., El Guedj, M., Vaz, T., Vantilcke, V., Mahamat, A., Magnien, C., Chauvet, E., Carme, B., Couppie, P., 2008. AIDS-related Histoplasma capsulatum var. capsulatum infection: 25 years experience of French Guiana. AIDS 22, 1047-1053.

Jones, O., Cleveland, K.O., Gelfand, M.S., 2009. A case of disseminated histoplasmosis following autologous stem cell transplantation for Hodgkin's lymphoma: an initial misdiagnosis with a false-positive serum galactomannan assay. Transpl. Infect. Dis. 11, 281-283.

Kauffman, C.A., 2007. Histoplasmosis: a clinical and laboratory update. Clin. Microbiol. Rev. 20, 115-132.

Kauffman, C.A., 2008. Diagnosis of histoplasmosis in immunosuppressed patients. Curr. Opin. Infect. Dis. 21, 421-425.

Lewden, C., Sobesky, M., Cabie, A., Couppie, P., Boulard, F., Bissuel, F., May, T., Morlat, P., Chene, G., Lamaury, I., Salmon, D., 2004. [Causes of death among HIV infected adults in French Guyana and the French West Indies in the era of highly active antiretroviral therapy (HAART)]. Medecine et maladies infectieuses 34, 286-292.

Moir, S., Fauci, A.S., 2009. B cells in HIV infection and disease. Nature reviews. Immunology 9, 235-245.

Narreddy, S., Chandrasekar, P.H., 2008. False-positive Aspergillus galactomannan (GM) assay in histoplasmosis. The Journal of infection 56, 80-81. 
Pineau, S., Talarmin, J.P., Morio, F., Grossi, O., Boutoille, D., Leaute, F., Le Pape, P., GayAndrieu, F., Miegeville, M., Raffi, F., 2010. [Contribution of molecular biology and Aspergillus galactomannan antigen assay for the diagnosis of histoplasmosis]. Med. Mal. Infect. 40, 541-543.

Ranque, S., Pelletier, R., Michel-Nguyen, A., Dromer, F., 2007. Platelia Aspergillus assay for diagnosis of disseminated histoplasmosis. Eur. J. Clin. Microbiol. Infect. Dis. 26, 941-943.

Riviere, S., Denis, B., Bougnoux, M.E., Lanternier, F., Lecuit, M., Lortholary, O., 2012. Serum Aspergillus galactomannan for the management of disseminated histoplasmosis in AIDS. Am. J. Trop. Med. Hyg. 87, 303-305.

Sathapatayavongs, B., Batteiger, B.E., Wheat, J., Slama, T.G., Wass, J.L., 1983. Clinical and laboratory features of disseminated histoplasmosis during two large urban outbreaks. Medicine 62, 263-270.

Simon, S., Veron, V., Boukhari, R., Blanchet, D., Aznar, C., 2010. Detection of Histoplasma capsulatum DNA in human samples by real-time polymerase chain reaction. Diagn. Microbiol. Infect. Dis. 66, 268-273.

Therby, A., Polotzanu, O., Khau, D., Monnier, S., Greder Belan, A., Eloy, O., 2014. [Aspergillus galactomannan assay for the management of histoplasmosis due to Histoplasma capsulatum var. duboisii in HIV-infected patients: Education from a clinical case]. J. Mycol. Med. 24, 166-170.

Tobon, A.M., Agudelo, C.A., Rosero, D.S., Ochoa, J.E., De Bedout, C., Zuluaga, A., Arango, M., Cano, L.E., Sampedro, J., Restrepo, A., 2005. Disseminated histoplasmosis: a comparative study between patients with acquired immunodeficiency syndrome and nonhuman immunodeficiency virus-infected individuals. Am. J. Trop. Med. Hyg. 73, 576-582.

Tong, K.B., Lau, C.J., Murtagh, K., Layton, A.J., Seifeldin, R., 2009. The economic impact of aspergillosis: analysis of hospital expenditures across patient subgroups. Int. J. Infect. Dis. $13,24-36$.

Van Der Veer, J., Lewis, R.J., Emtiazjoo, A.M., Allen, S.D., Wheat, L.J., Hage, C.A., 2012. Cross-reactivity in the Platelia Aspergillus enzyme immunoassay caused by blastomycosis. Med. Mycol. 50, 396-398.

Vergidis, P., Walker, R.C., Kaul, D.R., Kauffman, C.A., Freifeld, A.G., Slagle, D.C., Kressel, A.K., Wheat, L.J., 2012. False-positive Aspergillus galactomannan assay in solid organ transplant recipients with histoplasmosis. Transpl. Infect. Dis. 14, 213-217.

Wheat, L.J., 2006. Antigen detection, serology, and molecular diagnosis of invasive mycoses in the immunocompromised host. Transplant infectious disease : an official journal of the Transplantation Society 8, 128-139.

Wheat, L.J., Hackett, E., Durkin, M., Connolly, P., Petraitiene, R., Walsh, T.J., Knox, K., Hage, C., 2007. Histoplasmosis-associated cross-reactivity in the BioRad Platelia Aspergillus enzyme immunoassay. Clin. Vaccine Immunol. 14, 638-640.

Xavier, M.O., Pasqualotto, A.C., Cardoso, I.C., Severo, L.C., 2009. Cross-reactivity of Paracoccidioides brasiliensis, Histoplasma capsulatum, and Cryptococcus species in the commercial Platelia Aspergillus enzyme immunoassay. Clin. Vaccine Immunol. 16, 132133. 
TABLES

Table 1: Patients baseline characteristics, galactomannan and Histoplasma-specific antibody detection in AIDS patients with or without histoplasmosis

4

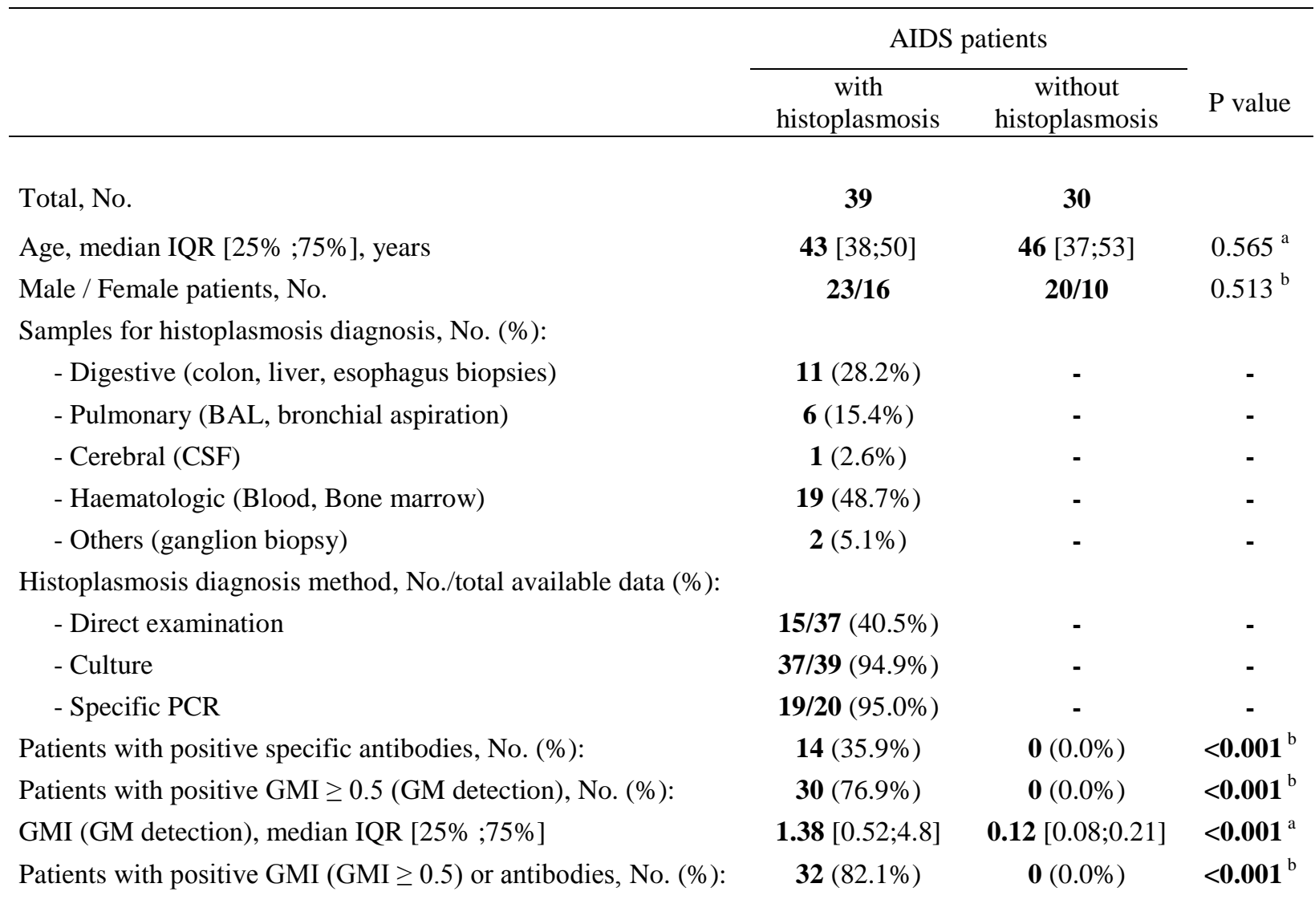

${ }^{\mathrm{a}}$ calculated by Mann-Whitney rank sum test; ${ }^{\mathrm{b}}$ calculated by $\chi^{2}$ test BAL: broncho-alveolar lavage, CSF: Cerebro-spinal fluid 
1 FIGURE LEGEND

2 Fig 1: Performance of GM detection according to the threshold

3 Receiver Operating Characteristics (ROC) curve for determination of cut-off and assay

4 sensitivity (Se) and specificity (Sp). The thresholds $0.35,0.4$ and 0.5 are shown on the ROC

5 curve with a triangle, circle and square, respectively.

6

7

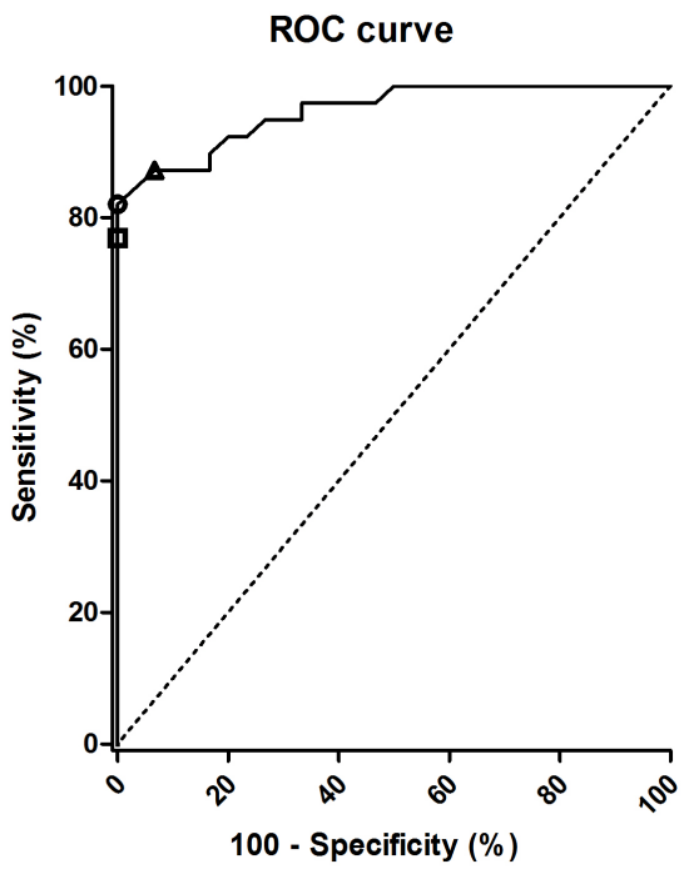

10

\begin{tabular}{ccc}
\hline & \multicolumn{2}{c}{ Performance } \\
\cline { 2 - 3 } $\begin{array}{c}\text { GMI } \\
\text { threshold }\end{array}$ & Se (\%) & Sp (\%) \\
\hline 0.10 & 100 & 36.7 \\
0.20 & 94.9 & 70,0 \\
0.30 & 87.2 & 90,0 \\
$\mathbf{0 . 3 5}$ & $\mathbf{8 7 . 2}$ & $\mathbf{9 3 . 3}$ \\
$\mathbf{0 . 4 0}$ & $\mathbf{8 2 . 1}$ & $\mathbf{1 0 0}$ \\
$\mathbf{0 . 5 0}$ & $\mathbf{7 6 . 9}$ & $\mathbf{1 0 0}$ \\
0.60 & 66.7 & 100 \\
2.00 & 33.3 & 100 \\
15.00 & 0,0 & 100 \\
\hline
\end{tabular}

9

100 - Specificity $(\%)$ 